\title{
Improved Newton-Raphson methods for Solving Nonlinear Equations
}

\author{
Liang Fang ${ }^{1, *}$, Lin Pang ${ }^{2}$ \\ ${ }^{1}$ College of Mathematics and Statistics, Taishan University, 271021, Tai'an, China \\ fangliang3@163.com \\ ${ }^{2}$ The circulation department of Library, Taishan University, 271021, Tai'an, China \\ panglin_lin@163.com
}

\section{ABSTRACT}

In this paper, we mainly study the numerical algorithms for simple root of nonlinear equations based on Newton-Raphson method. Two modified Newton-Raphson methods for solving nonlinear equations are suggested. Both of the methods are free from second derivatives. Numerical examples are made to show the performance of the presented methods, and to compare with other ones. The numerical results illustrate that the proposed methods are more efficient and performs better than Newton-Raphson method.

\section{Keywords}

Newton-Raphson method; Nonlinear equations; Iterative method; Order of convergence; Efficiency index.

\section{SUBJECT CLASSIFICATION}

Mathematics Subject Classification: 41A25, 65H05, 65D99

\section{Introduction}

Nonlinear problems is an important direction of research in the field of numerical calculation. Solving nonlinear equations is a classical problem which has interesting applications in various branches of science and engineering, and the problem of solving nonlinear equations by numerical methods has gained more importance than before.

To solve nonlinear equations, we can use iterative methods such as Newton's method (NM), Newton-Raphson method (NRM) or their variants.

In this paper, to improve the convergence properties, we consider iterative method to find a simple root $x *$ of a nonlinear equation $f(x)=0$, where $f: \Gamma \subseteq R \rightarrow R$ for an open interval $\Gamma$ is a scalar function and it is sufficiently differentiable in a neighborhood of $x^{*}$.

It is well known that Newton-Raphson method is a basic and important method for solving nonlinear equation [1] using the iterative expression

$$
x_{n+1}=x_{n}-\frac{f\left(x_{n}\right)}{f^{\prime}\left(x_{n}\right)}
$$

which is quadratically convergent in the neighborhood of $x^{*}$.

To improve the convergence properties, many variants of Newton-Raphson method has been proposed in the last years. In [2], Potra and Pták presented the following iterative method (PPM)

$$
x_{n+1}=x_{n}-\frac{f\left(x_{n}\right)+f\left(y_{n}\right)}{f^{\prime}\left(x_{n}\right)}
$$

which is cubically convergent. In [3], Changbum Chun presented the following iterative scheme (CM)

$$
x_{n+1}=x_{n}-\frac{f\left(x_{n}\right)}{f^{\prime}\left(x_{n}\right)}-\frac{2 f\left(y_{n}\right)}{f^{\prime}\left(x_{n}\right)}+\frac{f\left(y_{n}\right) f^{\prime}\left(y_{n}\right)}{f^{\prime 2}\left(x_{n}\right)}
$$

which is fourth-order convergent

In recent years, much attention has been given to develop iterative methods for solving nonlinear equations and a vast literature has been produced [3-10].

Motivated and inspired by the on going activities in this direction, the aim of this paper is to construct new efficient iterative methods to solve nonlinear equations. Based on Newton-Raphson method, we present two improved NewtonRaphson methods, and the convergence of the new scheme is proved. The proposed methods do require the computation of only first-order derivatives unlike other methods requiring the computation of second or higher derivatives. The order of convergence of the proposed algorithms is established. Several examples are presented and compared to other methods, showing the accuracy and fast convergence of the new algorithms.

\section{The Improved Newton-Raphson Methods And Their Convergence}

Let us consider the following iterative method.

Algorithm 1. For given $x_{0}$, we consider the following iteration scheme

$$
y_{n}=x_{n}-\frac{f\left(x_{n}\right)}{f^{\prime}\left(x_{n}\right)},
$$


(5)

$$
w_{n}=y_{n}-\frac{2 f\left(y_{n}\right)}{f^{\prime}\left(x_{n}\right)}+\frac{f\left(y_{n}\right) f^{\prime}\left(y_{n}\right)}{f^{\prime 2}\left(x_{n}\right)} \text {, }
$$

(6)

$$
x_{n+1}=w_{n}-\frac{3 f^{\prime 2}\left(x_{n}\right)+f^{\prime 2}\left(y_{n}\right)}{2 f^{\prime}\left(x_{n}\right) f^{\prime}\left(y_{n}\right)+2 f^{\prime 2}\left(y_{n}\right)} \frac{f\left(w_{n}\right)}{f^{\prime}\left(x_{n}\right)} .
$$

Now, we are in the position to give the convergence of Algorithm 1.

Theorem 1. Assume that the function $f: \Gamma \subseteq R \rightarrow R$ has a single root $x^{*} \in \Gamma$, where $\Gamma$ is an open interval. If $f(x)$ has first, second and third derivatives in $\Gamma$, then Algorithm 1 is sixth-order convergent in a neighborhood of $x *$ and it satisfies error equation

$$
e_{n+1}=5 c_{2}^{5} e_{n}^{6}+O\left(e_{n}^{7}\right)
$$

where

$$
e_{n}=x_{n}-x^{*}, c_{k}=\frac{f^{(k)}\left(x^{*}\right)}{k ! f^{\prime}\left(x^{*}\right)}, k=1,2, \cdots
$$

Proof. Let $x^{*} \in \Gamma$ be the simple root of $f(x)$,

$$
c_{k}=\frac{f^{(k)}\left(x^{*}\right)}{k ! f^{\prime}\left(x^{*}\right)}, k=1,2, \cdots \text { and } e_{n}=x_{n}-x^{*} .
$$

Consider the iteration function $F(x)$ defined by

$$
F(x)=w(x)-\frac{3 f^{\prime 2}(x)+f^{\prime 2}(y(x))}{2 f^{\prime}(x) f^{\prime}(y(x))+2 f^{\prime 2}(y(x))} \frac{f(w(x))}{f^{\prime}(x)}
$$

where

$$
w(x)=y(x)-\frac{2 f(y(x))}{f^{\prime}(x)}+\frac{f(y(x)) f^{\prime}(y(x))}{f^{\prime 2}(x)}, \quad y(x)=x-\frac{f(x)}{f^{\prime}(x)}
$$

By some computations using Maple we can obtain

$$
F\left(x^{*}\right)=x^{*}, F^{(i)}\left(x^{*}\right)=0, i=1,2,3,4,5, \quad F^{(6)}\left(x^{*}\right)=\frac{225 f^{(2)}\left(x^{*}\right)^{5}}{2 f^{\prime}\left(x^{*}\right)^{5}} \text {. }
$$

Furthermore, from the Taylor expansion of $F\left(x_{n}\right)$ around $x^{*}$, we have

$$
\begin{aligned}
x_{n+1}= & F\left(x_{n}\right)=F\left(x^{*}\right)+F^{\prime}\left(x^{*}\right)\left(x_{n}-x^{*}\right)+\frac{F^{(2)}\left(x^{*}\right)}{2 !}\left(x_{n}-x^{*}\right)^{2}+\frac{F^{(3)}\left(x^{*}\right)}{3 !}\left(x_{n}-x^{*}\right)^{3} \\
& +\frac{F^{(4)}\left(x^{*}\right)}{4 !}\left(x_{n}-x^{*}\right)^{4}+\frac{F^{(5)}\left(x^{*}\right)}{5 !}\left(x_{n}-x^{*}\right)^{5}+\frac{F^{(6)}\left(x^{*}\right)}{6 !}\left(x_{n}-x^{*}\right)^{6}+O\left(\left(x_{n}-x^{*}\right)^{7}\right) .
\end{aligned}
$$

Substituting (11) into (12) yields

$$
x_{n+1}=x^{*}+e_{n+1}=x^{*}+5 c_{2}^{5} e_{n}^{6}+O\left(e_{n}^{7}\right) .
$$

Therefore, we have 


$$
e_{n+1}=5 c_{2}^{5} e_{n}^{6}+O\left(e_{n}^{7}\right)
$$

which shows that the algorithm 1 is sixth-order convergent.

Now, we give the other further improved Newton-Raphson method.

Algorithm 2. For given $x_{0}$, we consider the following iteration scheme

$$
\begin{aligned}
& y_{n}=x_{n}-\frac{f\left(x_{n}\right)}{f^{\prime}\left(x_{n}\right)}, \\
& w_{n}=y_{n}-\frac{2 f\left(y_{n}\right)}{f^{\prime}\left(x_{n}\right)}+\frac{f\left(y_{n}\right) f^{\prime}\left(y_{n}\right)}{f^{\prime 2}\left(x_{n}\right)},
\end{aligned}
$$

(14)

$$
x_{n+1}=w_{n}+\frac{f^{\prime}\left(x_{n}\right)+f^{\prime}\left(y_{n}\right)}{f^{\prime}\left(x_{n}\right)-3 f^{\prime}\left(y_{n}\right)} \frac{f\left(w_{n}\right)}{f^{\prime}\left(x_{n}\right)} .
$$

Next, we consider the convergence of Algorithm 2.

Theorem 2. Assume that the function $f: \Gamma \subseteq R \rightarrow R$ has a single root $x^{*} \in \Gamma$, where $\Gamma$ is an open interval. If $f(x)$ has first, second and third derivatives in $\Gamma$, then Algorithm 2 is seventh-order convergent in a neighborhood of $x *$ and it satisfies error equation

$$
e_{n+1}=10 c_{2}^{4}\left(c_{2}^{2}-c_{3}\right) e_{n}^{7}+O\left(e_{n}^{8}\right)
$$

where

$$
e_{n}=x_{n}-x^{*}, c_{k}=\frac{f^{(k)}\left(x^{*}\right)}{k ! f^{\prime}\left(x^{*}\right)}, k=1,2, \cdots .
$$

Proof. Let $x^{*} \in \Gamma$ be the simple root of $f(x)$,

$$
c_{k}=\frac{f^{(k)}\left(x^{*}\right)}{k ! f^{\prime}\left(x^{*}\right)}, k=1,2, \cdots \text { and } e_{n}=x_{n}-x^{*} .
$$

Consider the iteration function $F(x)$ defined by

$$
F(x)=w(x)+\frac{f^{\prime}(x)+f^{\prime}(y(x))}{f^{\prime}(x)-3 f^{\prime}(y(x))} \frac{f(w(x))}{f^{\prime}(x)}
$$

where

$$
w(x)=y(x)-\frac{2 f(y(x))}{f^{\prime}(x)}+\frac{f(y(x)) f^{\prime}(y(x))}{f^{\prime^{2}}(x)}, \quad y(x)=x-\frac{f(x)}{f^{\prime}(x)}
$$

By some computations we can obtain

$$
\begin{aligned}
& F\left(x^{*}\right)=x^{*}, F^{(i)}\left(x^{*}\right)=0, i=1,2,3,4,5,6 \\
& F^{(7)}\left(x^{*}\right)=-\frac{225 f^{(4)}\left(x^{*}\right)\left[-3\left(f^{\prime 2}\left(x^{*}\right)+2 f^{(3)}\left(x^{*}\right) f^{\prime}\left(x^{*}\right)\right]\right.}{2 f^{\prime}\left(x^{*}\right)^{6}} .
\end{aligned}
$$

Furthermore, from the Taylor expansion of $F\left(x_{n}\right)$ around $x^{*}$, we have 


$$
\begin{aligned}
x_{n+1}= & F\left(x_{n}\right)=F\left(x^{*}\right)+F^{\prime}\left(x^{*}\right)\left(x_{n}-x^{*}\right)+\frac{F^{(2)}\left(x^{*}\right)}{2 !}\left(x_{n}-x^{*}\right)^{2}+\frac{F^{(3)}\left(x^{*}\right)}{3 !}\left(x_{n}-x^{*}\right)^{3} \\
& +\frac{F^{(4)}\left(x^{*}\right)}{4 !}\left(x_{n}-x^{*}\right)^{4}+\frac{F^{(5)}\left(x^{*}\right)}{5 !}\left(x_{n}-x^{*}\right)^{5}+\frac{F^{(6)}\left(x^{*}\right)}{6 !}\left(x_{n}-x^{*}\right)^{6} \\
& +\frac{F^{(7)}\left(x^{*}\right)}{7 !}\left(x_{n}-x^{*}\right)^{7}+O\left(\left(x_{n}-x^{*}\right)^{8}\right) .
\end{aligned}
$$

Substituting (18) into (19) yields

$$
x_{n+1}=x^{*}+e_{n+1}=x^{*}+10 c_{2}^{4}\left(c_{2}^{2}-c_{3}\right) e_{n}^{7}+O\left(e_{n}^{8}\right) .
$$

Therefore, we have

$$
e_{n+1}=10 c_{2}^{4}\left(c_{2}^{2}-c_{3}\right) e_{n}^{7}+O\left(e_{n}^{8}\right)
$$

which shows that the algorithm 2 is seventh-order convergent.

\section{Numerical Results}

Now, we employ Algorithm 1 and Algorithm 2 presented in this paper to solve some nonlinear equations and compare them with NRM and PPM. Displayed in Table 1 are the number of iterations (ITs) required such that $\left|f\left(x_{n}\right)\right|<1 . E-14$.

Table 1. Comparison of Algorithm 1, Algorithm 2, NRM PPM and CM

\begin{tabular}{ccccccc}
\hline Functions & $x_{0}$ & NRM & PPM & CM & Algorithm 1 & Algorithm 2 \\
\hline$f_{1}$ & 2.4 & 7 & 4 & 3 & 3 & 3 \\
& 1.6 & 6 & 12 & 5 & 4 & 4 \\
\hline$f_{2}$ & 0.6 & 4 & 3 & 3 & 3 & 2 \\
& 3.1 & 7 & 3 & 3 & 2 & 3 \\
\hline$f_{3}$ & 1.4 & 6 & 4 & 3 & 3 & 3 \\
& 2.1 & 5 & 4 & 4 & 3 & 3 \\
\hline$f_{4}$ & 3.8 & 16 & 12 & 8 & 7 & 6 \\
& 3.3 & 10 & 7 & 7 & 6 & 6 \\
\hline$f_{5}$ & 7.7 & 13 & 9 & 6 & 5 & 4 \\
& -0.8 & 6 & 5 & 4 & 4 & 3 \\
\hline
\end{tabular}

In table 1, we use the following functions.

$$
\begin{aligned}
& f_{1}(x)=(x-1)^{3}-1, x^{*}=2 . \\
& f_{2}(x)=\cos x-x, \alpha=0.73908513321516 . \\
& f_{3}(x)=\sin ^{2}(x)-x^{2}+1, x^{*}=1.40449164885154 . \\
& f_{4}(x)=e^{x^{2}+7 x-30}-1, x^{*}=3 .
\end{aligned}
$$




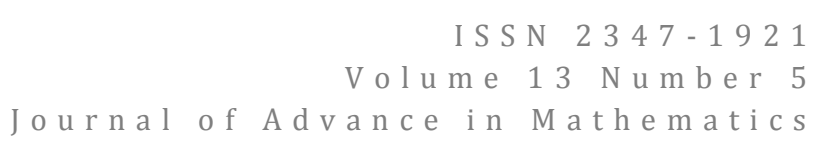

$f_{5}(x)=(x+2) e^{x}-1, x^{*}=-0.44285440096708$.

The computational results in Table 1 show that Algorithm 1 and Algorithm 2 require less ITs than NRM and PPM. Therefore, the present algorithms are of practical interest and can compete with NRM, PPM, CM and some existing algorithms.

\section{Conclusions}

With the wide development of science and technology, the problem of solving nonlinear equations by numerical methods has gained more importance than before. In order to obtain efficient algorithm for the nonlinear equations which come from the practical problems, in this paper, we present and analyze two improved Newton-Raphson methods for solving nonlinear equations. The methods are free from second derivatives. Several numerical results illustrate the convergence behavior and computational efficiency of the proposed methods. Computational results demonstrate that they are more efficient and perform better than Newton-Raphson method and some existing methods.

\section{Acknowledgement}

The work is supported by Project of Natural Science Foundation of Shandong province (ZR2016AM06), Excellent Young Scientist Foundation of Shandong Province (BS2011SF024).

\section{REFERENCES}

[1] Traub, J. F. 1964. Iterative Methods for Solution of Equations, Prentice-Hall, Englewood Clis, NJ.

[2] Potra, F.A., Potra-Pták. 1984. Nondiscrete induction and iterative processes, Research Notes in Mathematics, Vol. 103, Pitman, Boston.

[3] Chun, C. B. 2006. A new iterative method for solving nonlinear equations, Appl. Math. Comput. 178, p. 415-422.

[4] Fang, L., He, G. P., and Hu, Z. Y. 2008. A cubically convergent Newton-type method under weak conditions, J. Comput. Appl. Math. 220, p. 409-412.

[5] Kou, J. S. 2007. The improvements of modified Newton's method, Appl. Math. Comput. 189, p. 602-609.

[6] Abbasbandy, S. 2003. Improving Newton-Raphson method for nonlinear equations by modified Adomian decomposition method, Appl. Math. Comput. 145, p. 887-893.

[7] Fang, L. and He G. P. 2009. Some modifications of Newton's method with higher-order convergence for solving nonlinear equations, J. Comput. Appl. Math. 228, p. 296-303.

[8] Chun, C. B. 2008. A simply constructed third-order modifications of Newton's method, J. Comput. Appl. Math. 219, p. 81-89.

[9] Fang, L., Sun, L. and He G. P. 2008. An efficient Newton-type method with fifth-order for solving nonlinear equations, Comput. Appl. Math. 27, p. 269-274.

[10] Grau, M. and Diaz-Barrero, J.L. 2006. An improvement to Ostrowski root-finding method, Appl. Math. Comput. 173 , p. $450-456$.

\section{Authors' biography with Photo}

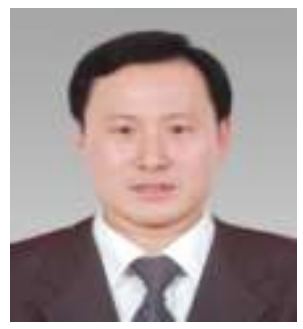

Liang Fang is a professor at Taishan University. He obtained his PhD from Shanghai Jiaotong University in June, 2010. His research interests are in the areas of cone optimization, and complementarity problems.

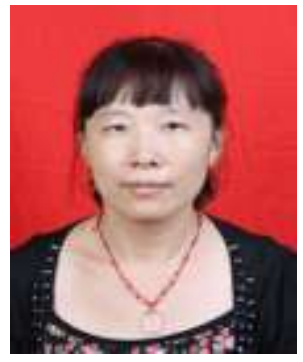

Lin Pang is a Librarian at Taishan University. She obtained her Bachelor degree from Taishan University in June, 2011. Her research interests are Computer science, Literature, etc. 